\title{
Research of Image Transmission System Based on ZigBee and GPRS Network
}

\author{
Xu Liu, Da Zhang, Xiao Lv, and Feng Jin
}

\begin{abstract}
It is urgent to need the rich information of the image etc. introduced into wireless sensor network based monitoring. This paper introduces an image transmission system based on wireless sensor networks using the ZigBee and GPRS network. The ZigBee network uses mesh network topology, which is simple and robust. To realize the remote transmission, this paper uses GPRS network, and it can realize seamless connection of the ZigBee Coordinator node, GPRS and the internet. Through the image transmission system, it can quickly transmit the image to the monitoring center, and the monitoring center can composite the image by the transmitted image data from ZigBee End-Device. Through the image transmission system, it can make up transmission data single by WSNs. It will have a fine application prospects in the image monitor system.
\end{abstract}

Index Terms-ZigBee, GPRS, image, WSNs, socket.

\section{INTRODUCTION}

Wireless sensor networks(WSNs)are widely used in industrial applications, such as environmental monitoring, surveillance, object tracking and health monitoring. At present, WSNs nodes are designed mainly for obtaining temperature, humidity, light intensity, etc. [1]. However, such simple data can't meet the full demands for large environmental monitoring and industrial applications.It is urgent to need the rich information of the image, audio, video and other multimedia introduced into the sensor network based monitoring.

Several work has been done on image transmission over WSNs. For example, Georgiy Pekhteryev etc. tested the JPEG and JPEG-2000 images over ZigBee networks showing that JPEG-2000 images encoded into multiple quality layers are more error-resilient, while maintaining a high peak signal-to-noise ratio (PSNR) [2]. Jaime Lloret et al. presented a WSNs in which each sensor node takes images from the field and uses internal image processing to detect any unusual status in the leaves [3].

It is important transmit images to a monitoring center through a WSNs.We can get the human activity information or the operating status of equipments.So it can provide more accurate monitoring of industrial and family careapplications.

In this paper the image transmission system uses ZigBee and GPRS network,it can quickly transmit the image to the monitoring center.

Manuscript received August 29, 2013; revised October 17, 2013.

The authors are with Beijing General Research Institute of Mining \&Metallurgy Beijing, China (e-mail: Liuxu1224@126.com).

\section{RELATED WORK}

\section{A. ZigBee Protocol Specification}

In the ZigBee network it inludes three types ofnetwork nodes, ZigBee Coordinator (Coordinator), ZigBeeRouter (Router) and ZigBee End-Device (terminal)respectively. ZigBee network consists of a Coordinator, multiple of the Routers and End-Devices. ZigBee coordinator shouldset up a new network features, and ZigBee routers andZigBeeEnd-Devices should provide support in a network. As shown in Fig. 1 is ZigBee wireless sensor network diagram.

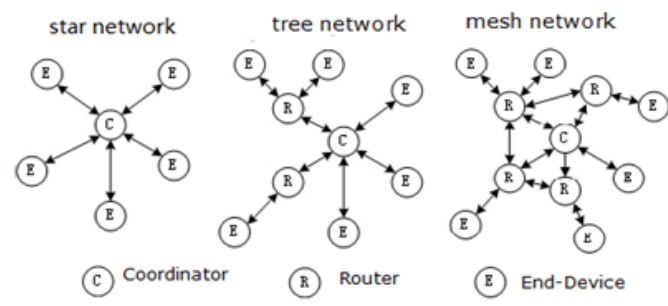

Fig. 1. Wireless sensor network diagram.

\section{B. ZigBee Protocol Stack and Runninganalysis}

Because of the ZigBee protocol stack has multiple versions of implementation, such as $\mathrm{Ti}$ Z-Stackof $\mathrm{Ti}$ company:ZigBee2004, ZigBee2006, ZigBee2007PRO, considering the system should have good practicability and reliability,so the wireless sensor network image transmission system adopts Z- Stack ZigBee2006. This protocol stack code uses most of $\mathrm{C}$ language to implement, the minority written by assembly language. ZigBee2006 protocol stack structure as shown in Fig. 2.

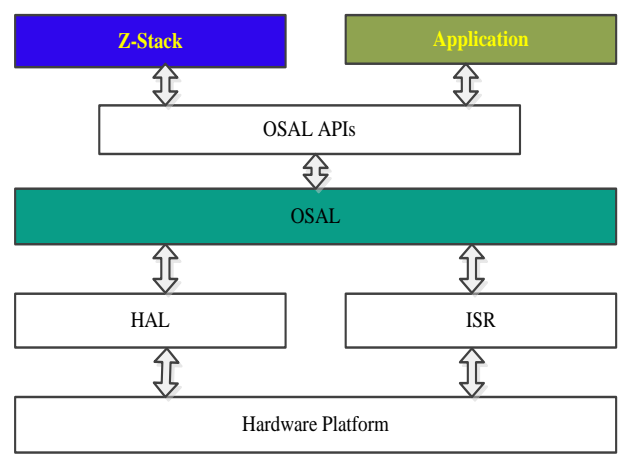

Fig. 2. ZigBee protocol stackarchitecture.

Z-Stack2006 protocol stack is based on the hardware abstraction layer (HAL), the operating system abstraction layer (OSAL), as well as through the OSAL management in the form of independent tasks reflect the concern of $\mathrm{ZigBee}$ protocol of each layer. OSAL provides the following 
services and management:task management, task synchronization, message management, time management, memory management, interrupt management, power management and the nonvolatile memory management .

Z-Stack2006 adopts the idea of operating system to build and useseventpolling mechanism, any of Z-Stack2006 subsystem as a task of OSAL.When after each layer initialized, the system to turn into low power mode. When there is a event trigger, it wake upfromlow power consumption in the system, and began to turn into the interrupt processing, after processing the continue to enter low power mode. If there are several events at the same time, it first judges of priorities of the events, and then successive processing the events.

Z-stack2006protocol stack running processes as shown in Fig. 3.

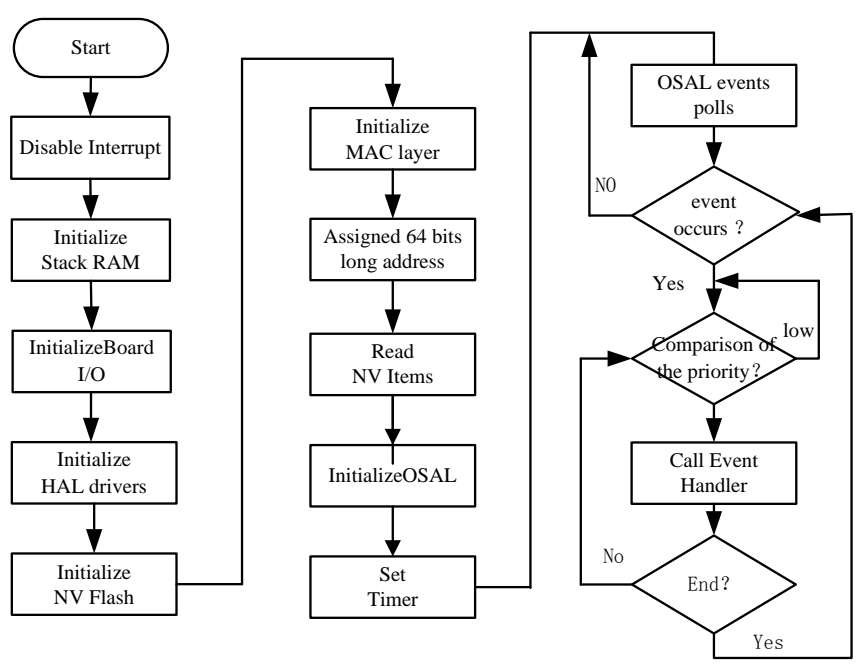

Fig. 3. Z - Stack system running flow chart.

\section{SYSTEM OVERVIEW}

\section{A. System Composition}

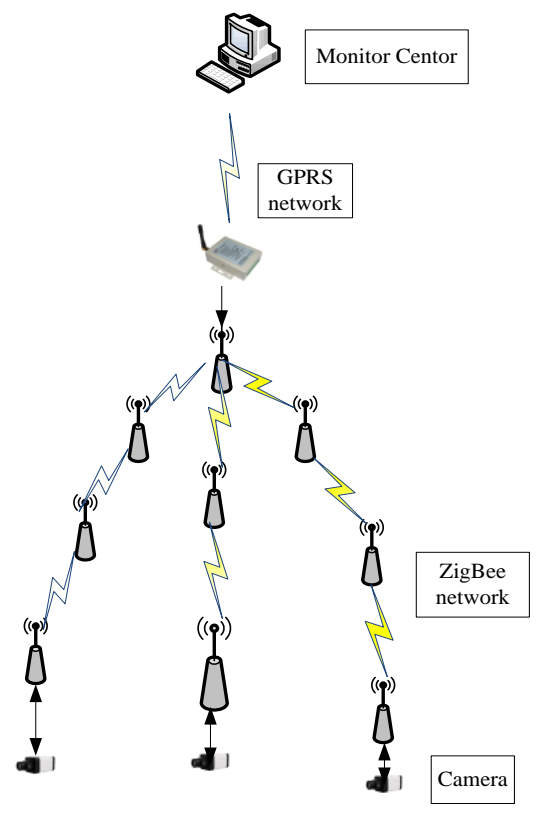

Fig. 4. The composition of the system.

The image transmission system is composed by ZigBee and GPRS network. In the system,there are an image acquisition terminals, a few ZigBee router nodes, aZigBee coordinator and GPRS module. Using GPRS technology to realize sending the image data to the remote monitoring center.The composition of the systemas shown in Fig. 4.

\section{B. Hardware Composition}

This image transmission system uses CC2430 chips of Ti company, on the whole chip, it integrates ZigBee RF front-end, memory and the microprocessor, and it uses 14 bits ADC. The chip has two serial ports,working at $2.4 \mathrm{GHz}$, and the data transmission rate is $250 \mathrm{Kbps}$.

The image acquisition node is composed of the ZigBee End-Device andcamera. We choose the camera moduleYSX-023.This type of camera has a standard RS-232 interface, which can be used with a PC or other equipment with RS232 interface.Wireless communication module is made up of chengdu dragon wireless communication technology co., LTD production of CC2430 chips, which is enhance performance type of $8051 \mathrm{MCU}$ processor module.

\section{Software Design}

\section{1) Wireless communication design}

The wireless transmission software is based on a ZigBee network, the network application was written in $\mathrm{C}$ and designed to run on a CC2430 system-on-chip (SoC) solution specifically tailored for IEEE 802.15.4 and ZigBee applications [4]-[8].

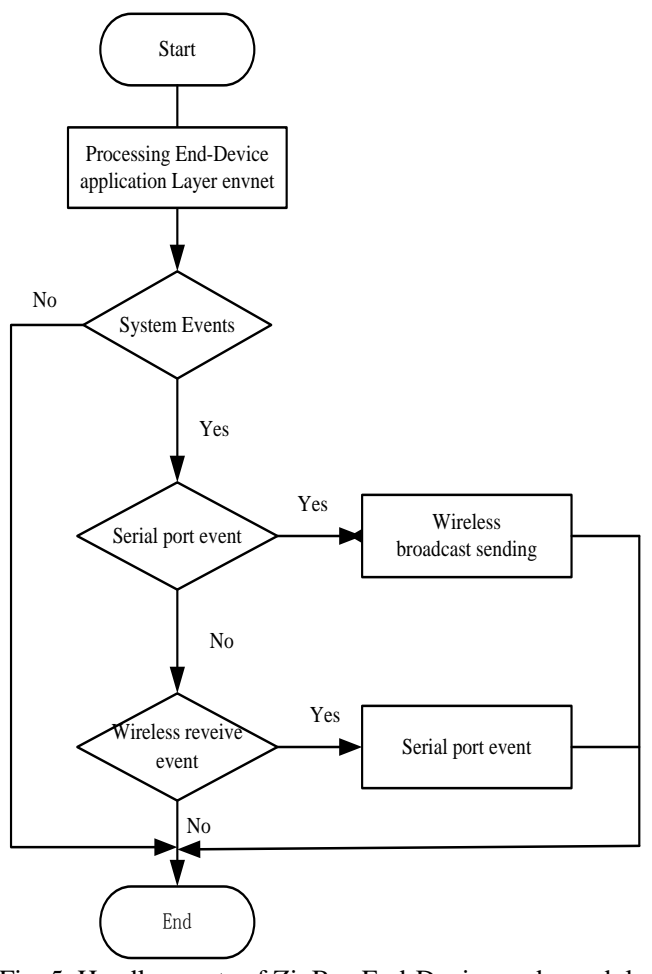

Fig. 5. Handle events of ZigBee End-Device node module.

The ZigBee Coordinator connects to the GPRS module. The main processing for ZigBeeCoordinatoris from the GPRS module of serial port application layer event handling and images from theZigBee End-Device node transmission data wireless sending and receiving process events. Also in the application level of the ZigBee End-Device node is mainly processing data from camera module serial port eventhandling and image data from the coordinator of 
wireless sending and receiving process events. Handle events of ZigBee End-Devicenode module as shown in Fig. 5 .

\section{2) GPRS connected to Internet communication with monitor center using Socket mechanism}

The image monitoring center uses Socket network communication mechanism, the monitoring center is responsible for monitoring the fixed ports, through sending commands and receiving that sent to the image data in the ZigBee wireless sensor network by remote GRPS DTU [9], [10].

The Socket server monitoring module workflow is as follows:

1) Through the IP Address and IP End-Point building IP End-Point instance to define the host object.

2) Through the Socket class to create a server Socket (Socket);

3) Through the Socket class to Bind () method to Bind the host information;

4) Through the Socket class Listen () method to set the ports for connection;

5) Through the Socket class the Accept () method to accept the client's connection request;

6) Through the Socket class method Receive()/the Send()to implement sending/receiving data;

7) To close the Socket.

The client working process is as follows:

1) To create user Socket (Socket);

2) To connect to a remote server (Connect), if accepted, to create the receiving process;

3) To start (Send/Receive data transmission);

4) To close the Socket (Close the Socket).

The Whole server and client work process is shown in Fig. 6.

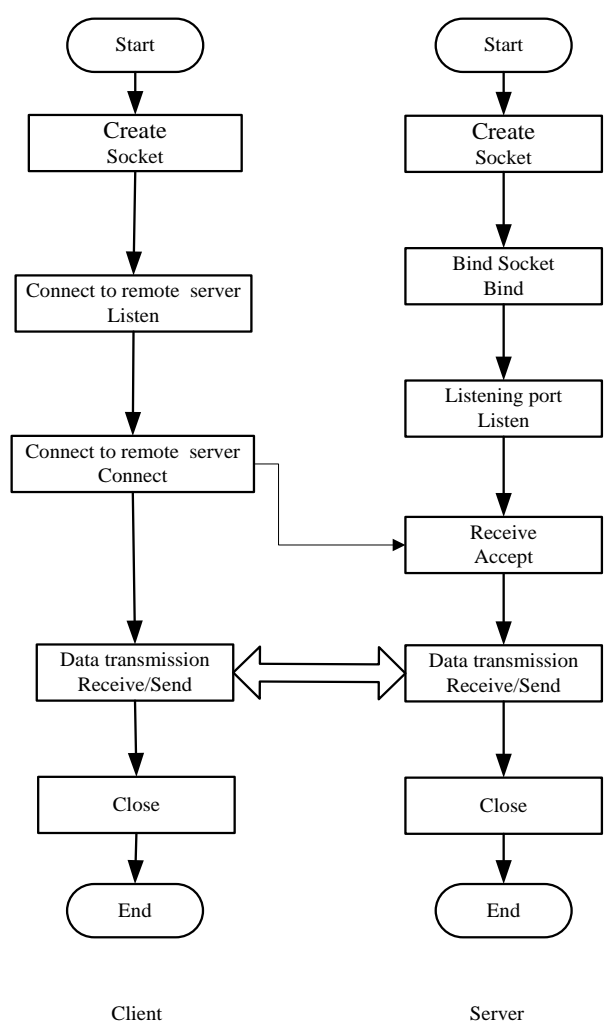

Fig. 6. The Whole server and client work process.

\section{3) Monitoring center software}

The monitoring center softwarehas four main functions:image acquisition module, image display module, image processing module, image storage module, the whole monitoring center software frame diagram as shown in Fig. 7.

Remote image acquisition module:the image information acquisition.

Image processing module: the acquisition of image information of synthetic images.

Image display module: the image for display.

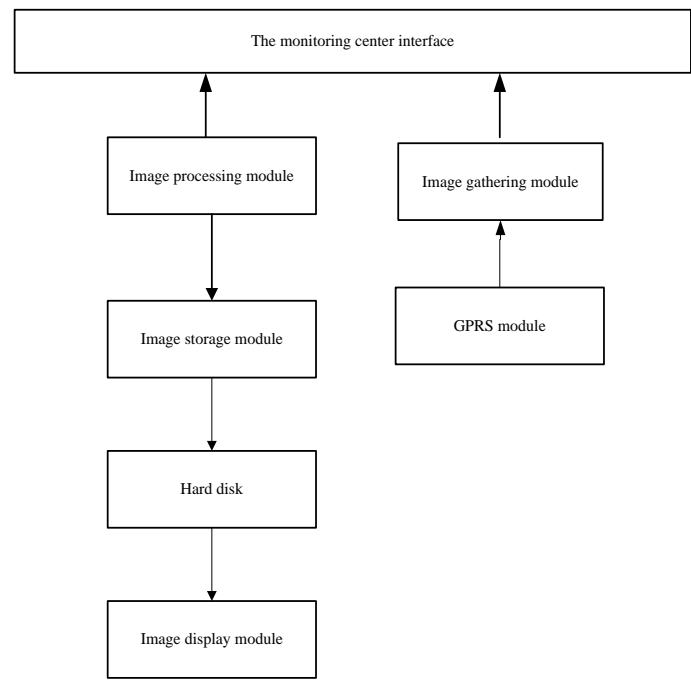

Fig. 7. Monitoring center system software frame diagram.

Above all, we introduce composition of the system based on wireless sensor networks using the ZigBee and GPRS network. Through the image transmission system, we can get image from remote monitoring point.

\section{CONCLUSION}

This paper introduces an image transmission system based on wireless sensor networks using the ZigBee and GPRS network. It presents the system composition, which includes hardware composition and software design. Through the image transmission system, it can make up transmission data single by WSNs. It will have fine application prospects in the image monitor system.

\section{ACKNOWLEDGMENT}

This research was supported by the National High Technology Research and Development Program of China (863 Program, Grant 2011AA060406) and International Cooperation Project (Grant 2011DFA71990).

\section{REFERENCES}

[1] H. F. Han, K. M. Du, Z. F. Sun, W. Zhao, R. Chen, and B. J. Liang, Trans. of the CSAE, vol. 25, pp. 158, 2009.

[2] G. Pekhteryev, Z. Sahinoglu, P. Orlik, and G. Bhatti., "Image transmission over IEEE 802.15.4 and ZigBee networks," Circuits and Systems, vol. 4, pp. 3539-3542, 2005.

[3] J. Lloret, I. Bosch, S. Sendra, and A. Serrano, "A wireless sensor network for vineyard monitoring that uses image processing," Sensors, vol. 11, no. 6, pp. 6165-6196, 2011.

[4] Y. L. Shi, Z. Y. Wang, X. Liu, D. J. Zhao, and L. Huang, "A web-based monitoring system as a measurement tool in greenhouse 
using wireless sensor network," Computer and Computing Technologies in Agriculture IV, vol. 346, pp. 289-297, 2009.

[5] J. Vana and B. Vedran, "Reducing power consumption of image transmission over IEEE 802.15.4/ZigBee sensor network," in Proc. 2010 IEEE International Instrumentation and Measurement Technology Conference, 2010

[6] H. Y. K. Yeon and R. S. Dong, "The security monitoring system using IEEE 802.15.4 protocol and CMOS Image Sensor," in Proc 2009 International Conf. on New Trends in Information and Service Science, 2009.

[7] P. S. Boluk, S. Baydere, and A. E. Harmanci, "Robust image transmission over wireless sensor networks," Mobile Networks and Applications, vol. 16, no. 2, pp. 149-170, 2011.

[8] Z. Q. Song, X. Z. Zhou, and L. Wang, "Research on wireless image transmission based on ZigBee," Computer Applications and Software vol. 29, no. 7, pp. 1-5, 2012

[9] Y. H. Kwon and D. R. Shin, "The security monitoring system using IEEE 802.15.4 protocol and CMOS Image Sensor," New Trends in Information and Service Science, IEEE Press, Beijing, 2009.

[10] S. C. Ma, M. Zhu, and L. Wang, "A remote monitoring system of IDC room based on ZigBee wireless sensor networks," presented a 2009 Eighth IEEE International Conference on Dependable, Autonomic and Secure Computing, Chengdu, 2009.

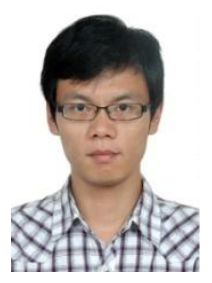

Xu Liu was born in Hebei, China on December 24, 1986. In 2010, he received the bachelor degrees from China Agricultural University, Beijing in electronic information science and technology. He received the master degree in computer science and technology from China Agricultural University, Beijing in 2012.

Currently, he is working in Beijing General Research Institute of Mining \& Metallurgy. He researches on mine wireless communication. He is now participating in projects "Ubiquitous information collection and mine wireless communication in underground metal" which is supported by the National 863 Project.
Da Zhang was born in Yingkou. In 2009, he received the doctor's degrees from northeastern university.

Currently, he is working in Beijing General Research Institute of Mining \& Metallurgy.

Xiao Lv was born in Hebei. In 2005, she received the bachelor degrees from South east University in Information \& Communication Engineering.

Currently, she is working in Beijing General Research Institute of Mining \& Metallurgy.

Feng Jin was born in Heilong Jiang. In 2008, he received the bachelor degrees from Harbin Institute of Technology. He received the master degree in Control Science and Engineering from Xi'an Jiaotong University in Xi'an, in 2011.

Currently, he is working in Beijing General Research Institute of Mining \& Metallurgy. 